Rep. Math. Phys.

\title{
Efficient quantum tomography needs complementary and symmetric measurements
}

\author{
Dénes Petz 1 and László Ruppert 2 \\ Alfréd Rényi Institute of Mathematics \\ H-1053 Budapest, Reáltanoda u. 13-15, Hungary \\ Department for Mathematical Analysis, \\ Budapest University of Technology and Economics \\ H-1521 Budapest XI., Hungary
}

\begin{abstract}
In this study the determinant of the average quadratic error matrix is used as the measure of state estimation efficiency. Minimizing this quantity gives us the optimal measurements in different scenarios. We present applications when von Neumann measurements or a single POVM are used, when there is no known information or a part of the parameters of the state is given. Under some restrictions the optimality is found for $n$-level systems. The optimal measurements have some complementary relation to each other and to the available data, moreover, symmetric informationally complete systems appear, containing a new, conditional version.
\end{abstract}

Key words and phrases: experiment design, state estimation, complementarity, measurement, quadratic error, qubit, symmetric informationally complete POVM.

\footnotetext{
${ }^{1}$ E-mail: petz@math.bme.hu.

${ }^{2}$ E-mail: ruppertl@math.bme.hu.
} 


\section{Introduction}

State estimation or tomography is a fundamental problem in the field of quantum information theory and it can be considered as one of the foundational issues of quantum mechanics [4, 17]. The topic of quantum tomography consists of methods which reconstruct the state of the system under investigation using repeated measurements of a set of observables. The problem may be traced back to the seventies [11], the interest in a thorough mathematical analysis of the quantum state estimation procedures has been flourishing recently [2, 10, 13, 25].

In statistics the accuracy of the estimation can be quantified by the quadratic error matrix. The matrices are typically not comparable by the positive semi-definiteness, hence if different estimation schemes are compared, the determinant of mean quadratic error matrix can be used instead. This approach can be found in references [19, 20], their main result was that the complementary von Neumann measurements are optimal. A more general context appears in [3], which is much closer to our approach. The concept of complementarity was extended to quantum subsystems in [21] and the case of two qubits has a detailed analysis in [14, 24]. When all parameters of the density matrix are obtained from a single measurement, then a symmetric informationally complete POVM appears [26]. A similar result was obtained earlier by Wootters and Fields [29] for von Neumann measurements and by Scott [28] for POVMs, but optimality had a different formulation in both cases.

Some a priori information about the state can be given in various ways, for example one can minimize the relative entropy to an a priori given state, resulting in a bias toward that state [15]. The most popular subject in this field is state discrimination: when we know that the system is one of several given states and we should figure out which one [5]. Beside knowing the possible states we can have an a priori probability distribution on the true state, too. This idea was used in [6] to obtain the optimal phase estimation. The given states do not construct a discrete set, instead, they are searching among all pure states. Another continuous subset is used in [12, where we know that the state to estimate is a convex combination of two given states. In our setup we know that the state is on a given subset of the whole state space (this can be the whole state space itself) and assume an arbitrary unitarily invariant a priori probability distribution to be known.

We use the determinant of the average quadratic error matrix as a figure of merit in state estimation setup; the average is taken over the unitarily invariant states. This quantity is relies on purely classical statistics, therefore it is much easier to compute than, e.g., quantum Fisher information, but still reproduces the well-known quantum phenomena and even more. So it gives us a useful tool to find the optimal measurement setup for different quantum tomography problems. We examine the assumption that the unknown state is known on some complementary subalgebras, which practically means that one should restrict the are of integration. For our calculations we use a parametrization for both the density operators and the observables (we expand them 
in a fixed basis that is orthonormal with respect to the Hilbert-Schmidt inner product), but we can see that the results are independent of this parametrization. To gain analytic results, some sort of symmetry is recommended but by using numerical methods we could overcome that and solve difficult problems efficiently.

Section 2 is a very short introduction, quantum states, measurements, state estimation, complementarity and symmetric measurements are overviewed. In Section 3, we give the motivation for using the determinant of the average covariance matrix by guiding through a simple example, and then give some useful remarks about this quantity. Then the optimization problem is solved for arbitrary dimensions under some restrictions. In Section 4 the complementarity of optimal von Neumann measurements is obtained. In Section 5 a single POVM is used for the measurement, then a symmetric informationally complete POVM appears. Finally, we investigate the asymmetrical case for POVMs too (Sec. 6) and draw conclusions (Sec. 7).

\section{Basic concepts}

In this section, we give a brief summary of the basic concepts appearing in the present paper (for further details, see [4, 17]).

$\rho \in M_{n}(\mathbb{C})$ is an n-dimensional quantum state if

$$
\rho \geq 0 \quad \text { and } \quad \operatorname{Tr} \rho=1 \text {. }
$$

We will use a basis of self-adjoint matrices to obtain a parametrization of these density matrices. In the qubit case, we use the Pauli matrices as a basis, i.e., the Bloch parametrization:

$$
\rho=\frac{1}{2}\left(I+\sum_{i=1}^{3} \theta_{i} \sigma_{i}\right)=\frac{1}{2}(I+\theta \cdot \sigma)=\frac{1}{2}\left[\begin{array}{cc}
1+\theta_{3} & \theta_{1}-\mathrm{i} \theta_{2} \\
\theta_{1}+\mathrm{i} \theta_{2} & 1-\theta_{3}
\end{array}\right] .
$$

In this case the whole state space of quantum states can be described using the Bloch vector: $\theta=\left(\theta_{1}, \theta_{2}, \theta_{3}\right)$ and the conditions in (1) are simply converted into $\theta_{1}^{2}+\theta_{2}^{2}+\theta_{3}^{2} \leq 1$.

For the n-dimensional case we use the following parametrization:

$$
\rho=\frac{1}{n}(I+\theta \cdot \sigma)
$$

where $\theta \in \mathbb{R}^{n^{2}-1}$ is the generalized Bloch vector, $\theta \cdot \sigma=\sum_{j} \theta_{j} \sigma_{j}$ and $\left\{\sigma_{j}: 1 \leq j \leq\right.$ $\left.n^{2}-1\right\}$ are generalized Pauli matrices, which form an orthonormal basis on the selfadjoint traceless matrices: $\sigma_{i}=\sigma_{i}^{*}, \operatorname{Tr} \sigma_{i}=0$ and $\operatorname{Tr} \sigma_{i} \sigma_{j}=\delta_{i, j}$. An example for the 3 -dimensional case are the multiples of Gell-Mann matrices. 
A positive operator-valued measure (POVM) can in this framework be described as a set of matrices:

$$
E=\left\{E_{1}, E_{2}, \ldots E_{k}\right\} \quad \text { with } \quad \sum_{i=1}^{k} E_{i}=I, E_{i}>0
$$

Von Neumann measurements can be described as a POVM with two components and projective elements: $\left\{P_{1}, I-P_{1}\right\}$.

Having a quantum state $\rho$ and a POVM $E$, the probability of obtaining an outcome related to the POVM element $E_{i}$ is:

$$
p_{i}=\operatorname{Tr}\left(\rho E_{i}\right) .
$$

If we have $m$ samples of the state $\rho$ and we measure $E$ on each copy independently, then let $\nu_{i}$ be the relative frequency of measuring the $i$ th outcome. If $m$ is a large number, then $\nu_{i}$ will be close to $p_{i}$, and if we know the POVM (because we set it), then we can make an estimation on $\rho$ from (3) by using $\nu_{i}$ instead of $p_{i}$. A simple state estimation problem is described in detail in Section 3 .

The heuristic concept of complementarity was born together with quantum theory. A mathematical definition is due to Accardi [1] and Kraus [9]. Let $\mathcal{H}$ be an $n$ dimensional Hilbert space. Let the observables $A$ and $B$ have eigenvectors $e_{1}, e_{2}, \ldots, e_{n}$ and $f_{1}, f_{2}, \ldots, f_{n}$ which are orthonormal bases, they are complementary if

$$
\left|\left\langle e_{i}, f_{j}\right\rangle\right|^{2}=\frac{1}{n} \quad(1 \leq i, j \leq n) .
$$

If this condition holds then the two bases are also called mutually unbiased. An overview about complementarity can be found in [22] and details are in [21, 24].

Complementarity can be generalized to the case of POVMs. The POVMs $\left\{E_{1}, E_{2}\right.$, $\left.\ldots, E_{k}\right\}$ and $\left\{F_{1}, F_{2}, \ldots, F_{m}\right\}$ are complementary if

$$
\operatorname{Tr} E_{i} F_{j}=\frac{1}{n} \operatorname{Tr} E_{i} \operatorname{Tr} F_{j} \quad(1 \leq i \leq k, \quad 1 \leq j \leq m) .
$$

This is equivalent to the orthogonality of the traceless parts:

$$
E_{i}-\frac{\operatorname{Tr} E_{i}}{n} I \quad \perp \quad F_{j}-\frac{\operatorname{Tr} F_{j}}{n} I,
$$

so we will use the expression quasi-orthogonal, when we use this property of complementary operators.

The concept can be extended to subalgebras $\mathcal{A}_{1}, \mathcal{A}_{2} \subset M_{n}(\mathbb{C}) . \quad \mathcal{A}_{1}$ and $\mathcal{A}_{2}$ are complementary if $A_{1} \in \mathcal{A}_{1}$ and $A_{2} \in \mathcal{A}_{2}$ are quasi-orthogonal (that is, the traceless part of matrices are orthogonal).

An orthonormal basis corresponds to the subalgebra of operators which are diagonal in this basis. Such a subalgebra is maximal Abelian, it will be called M-subalgebra. 
A symmetric informationally complete POVM $\left\{E_{i}: 1 \leq i \leq k\right\}$ of an $n$-level system is described by a set of projections $P_{i}(1 \leq i \leq k)$ such that

$$
\sum_{i=1}^{k} P_{i}=\lambda I \quad \text { and } \quad \operatorname{Tr} P_{i} P_{j}=\mu \quad(i \neq j),
$$

where $k=n^{2}, \lambda=n, \mu=1 /(n+1)$ and all projections are rank-one, see [26, 27]. The existence of a symmetric informationally complete POVM (SIC POVM) is not known for every dimension $n$.

In this paper $k<n^{2}$ case will also appear, when some parameters are already known, we need to estimate fewer parameters, i.e., fewer elements in POVM are needed to make a full reconstruction. If they fulfill condition (6) with some constants and the $P_{i \text {-s }}$ are complementary to the known subalgebra, then we call them conditional symmetric informationally complete POVM toward the known $n^{2}-k$ parameters, because they are informationally complete only if we use the condition that we want to discriminate the states only in the unknown directions. They include the case of SIC-POVMs as a special case when no parameters are known. The existence of such a POVM can be a fundamental question in different quantum tomography problems.

\section{The determinant of the average covariance ma- trix}

Suppose that we have a qubit and some of the three parameters $\theta_{1}, \theta_{2}$ and $\theta_{3}$ are known and the unknown parameters will be estimated. For example, let us assume that $\theta_{1}$ and $\theta_{2}$ are known and we want to estimate $\theta_{3}$. The assumption means that the reduced state is known on the M-subalgebras generated by $\sigma_{1}$ and $\sigma_{2}$, respectively. They are complementary subalgebras and the M-subalgebra generated by $\sigma_{3}$ is complementary to both. A projection of a von Neumann measurement

$$
E=\frac{1}{2}(I+\lambda \cdot \sigma)
$$

is used to estimate $\theta_{3}$ from the result of several measurements, where $\lambda_{1}^{2}+\lambda_{2}^{2}+\lambda_{3}^{2}=1$. The expected value is

$$
\operatorname{Tr} \rho E=\frac{1}{2}\left(1+\sum_{i=1}^{3} \theta_{i} \operatorname{Tr} \sigma_{i} E\right)=\frac{1}{2}(1+\langle\theta, \lambda\rangle)
$$

and we have

$$
\theta_{3} \operatorname{Tr} \sigma_{3} E=2 \operatorname{Tr} \rho E-\left(1+\sum_{i=1}^{2} \theta_{i} \operatorname{Tr} \sigma_{i} E\right) .
$$


Let us denote by $\nu$ the random outcome of the measurement of $E$, the expected value is $p=\operatorname{Tr} \rho E$ and it is Bernoulli distributed. The natural unbiased estimate of $\theta_{3}$ is

$$
\hat{\theta}_{3}=\frac{1}{\operatorname{Tr} \sigma_{3} E}\left(2 \nu-1-\sum_{i=1}^{2} \theta_{i} \operatorname{Tr} \sigma_{i} E\right) .
$$

The variance is

$$
\operatorname{Var}\left(\hat{\theta}_{3}\right)=\frac{4 p(1-p)}{\lambda_{3}^{2}}=\frac{1-\langle\lambda, \theta\rangle^{2}}{\lambda_{3}^{2}} .
$$

We want to argue that the optimal estimate corresponds to the projection $\lambda_{1}=$ $\lambda_{2}=0, \quad \lambda_{3}= \pm 1$, i.e. measuring in the unknown direction. This estimator has the variance $1-\theta_{3}^{2}$. However, the inequality

$$
\frac{1-\langle\lambda, \theta\rangle^{2}}{\lambda_{3}^{2}} \geq 1-\theta_{3}^{2}
$$

is not true in general, for example $\lambda=\theta$ is possible for pure states and then the lefthand side is zero. So if we want to minimize $\operatorname{Var}\left(\hat{\theta}_{3}\right)$, it is not true that $\frac{I+\sigma_{3}}{2}$ is the best observable.

Since $\theta_{1}, \theta_{2}$ are given, the only unitarily invariant vector to $\left(\theta_{1}, \theta_{2}, \theta_{3}\right)$ is $\left(\theta_{1}, \theta_{2},-\theta_{3}\right)$, if we take the average of the left hand side of (9) on these states we obtain

$$
\left\langle\operatorname{Var}\left(\hat{\theta}_{3}\right)\right\rangle=\frac{1}{2} \cdot \frac{1-\left(\lambda_{1} \theta_{1}+\lambda_{2} \theta_{2}+\lambda_{3} \theta_{3}\right)^{2}}{\lambda_{3}^{2}}+\frac{1}{2} \cdot \frac{1-\left(\lambda_{1} \theta_{1}+\lambda_{2} \theta_{2}-\lambda_{3} \theta_{3}\right)^{2}}{\lambda_{3}^{2}} .
$$

We can solve the minimization problem

$$
\left\langle\operatorname{Var}\left(\hat{\theta}_{3}\right)\right\rangle \rightarrow \min , \quad \lambda_{1}^{2}+\lambda_{2}^{2}+\lambda_{3}^{2}=1
$$

using the Schwarz inequality, and get that in the optimal case $\lambda_{1}=\lambda_{2}=0, \lambda_{3}= \pm 1$. Thus, the measurement of $\frac{I+\sigma_{3}}{2}$ is optimal indeed, yet only in the average sense.

Note that in (7) $\nu$ has Bernoulli distribution, with $p(1-p)$ variance. If $m$ measurements are performed, we will have $\nu=\left(\nu_{1}+\nu_{2}+\ldots+\nu_{m}\right) / m$, where $\nu_{i}$ is the outcome of the $i$-th measurement. Then the variance will be $\frac{m p(1-p)}{m^{2}}=\frac{1}{m} p(1-p)$, so we can conclude that if we have multiple measurements the variance changes only by a constant factor, hence the minimization problem remains unaffected.

In the following sections, we will always use the same kind of naturally derived estimators; one can prove that those are unbiased and efficient. We should also always bear in mind that if we have only a few measurements, we can easily get a Blochvector as a result of the estimation, which does not satisfy the positivity condition in (1). But in practical cases, we have many measurements, hence the law of large numbers ensures us that the estimator will give a state near the original one, and the probability of getting a physically impossible state (a density matrix with at least one 
negative eigenvalue) will converge to zero exponentially, due to the large deviation theory. That is why we do not have to deal with any physical restrictions, even if we calculate the variance only for one measurement in each case.

If we have multiple parameters, we can still get a multidimensional estimator from the measurement outcomes, but in that case, a covariance matrix will take the role of the variance. The average covariance matrix can be calculated by taking the piecewise average of the elements, but since the matrices are not comparable in general, we need an $M_{n}(\mathbb{C}) \rightarrow \mathbb{R}$ function which gives us a number as the objective function to minimize. After examining some candidates we concluded that the best choice is to take the determinant of the matrix and that this needs to be done after the averaging process, so we have the following optimization problem:

$$
\operatorname{det}\langle\operatorname{Cov}(\underline{\hat{\theta}})\rangle \rightarrow \min
$$

where $\underline{\hat{\theta}}$ is the estimator of the unknown parameters. This way we obtain an easily calculable quantity, and by solving the minimization problem, the optimal measurements.

If we want to take the average of this covariance matrix on the whole state space, we have to give a unitarily invariant measure on the state space. On the other hand, if we integrate on the unitarily invariant states of an initial state with respect to the normalized Haar measure and the minimum is independent of the initial state, we get optimal measurements independently from the (unitarily invariant) probability measure. Thus, we can obtain a stronger result than we would by using a particular measure.

Finally, let us note that using a POVM instead of von Neumann measurements is not a crucial difference. If there are $n$ unknown parameters, we need to have $n$ independent positive operators in the POVM. We only have the restriction $\sum E_{i}=I$, so $n+1$ operators are sufficient and we can construct the estimator from the outcomes related to the first $n$ components. The only difference is that in this case, the $n$ outcomes would not be independent, off-diagonal elements appear in the covariance matrix, too.

\section{The optimal von Neumann measurement}

The $n$-level quantum system is described by the algebra $M_{n}(\mathbb{C})$. Consider the following decomposition

$$
M_{n}(\mathbb{C})=\mathbb{C} I \oplus \mathcal{A} \oplus \mathcal{B},
$$

where $\mathcal{A}$ and $\mathcal{B}$ are linear subspaces and orthogonality is defined with respect to the Hilbert-Schmidt inner product $\langle A, B\rangle=\operatorname{Tr} A^{*} B$.

A state has the density matrix

$$
\rho=I / n+\rho_{\mathcal{A}}+\rho_{\mathcal{B}}
$$


We assume that the component $\rho_{\mathcal{B}}$ should be estimated. Let the dimension of $\mathcal{B}$ be $k$. The positive contractions $E^{1}, \ldots, E^{k}$ are used for independent measurements (on several identical copies of the $n$-level system): A measurement corresponds to the $\operatorname{POVM}\left\{E^{i}, I-E^{i}\right\}$. These operators have the expansion

$$
E^{i}=e_{i} I+E_{\mathcal{A}}^{i}+E_{\mathcal{B}}^{i} \quad(1 \leq i \leq k) .
$$

The expectations are

$$
p_{i}:=\operatorname{Tr} \rho E^{i}=e_{i}+\operatorname{Tr} \rho_{\mathcal{A}} E_{\mathcal{A}}^{i}+\operatorname{Tr} \rho_{\mathcal{B}} E_{\mathcal{B}}^{i} \quad(1 \leq i \leq k) .
$$

We fix an orthonormal basis $F_{1}, \ldots, F_{k}$ in $\mathcal{B}$. The unknown component has the expansion

$$
\rho_{\mathcal{B}}=\theta_{1} F_{1}+\ldots+\theta_{k} F_{k},
$$

where $\theta=\left(\theta_{1}, \ldots, \theta_{k}\right)$ are the parameters to be estimated. Similarly,

$$
E_{\mathcal{B}}^{i}=e_{i 1} F_{1}+e_{i 2} F_{2}+\ldots+e_{i k} F_{k}
$$

is an orthogonal expansion.

The estimates $\hat{\theta}_{i}$ are solutions of the equations

$$
\varepsilon_{i}=e_{i}+\operatorname{Tr} \rho_{\mathcal{A}} E_{\mathcal{A}}^{i}+\sum_{j=1}^{k} e_{i j} \hat{\theta}_{j},
$$

where $\varepsilon_{i}$ is the random result of the $i$ th measurement, $1 \leq i \leq k$. In another form

$$
\left[\begin{array}{c}
\varepsilon_{1} \\
\vdots \\
\varepsilon_{k}
\end{array}\right]=\left[\begin{array}{c}
e_{1} \\
\vdots \\
e_{k}
\end{array}\right]+\left[\begin{array}{c}
\operatorname{Tr} \rho_{\mathcal{A}} E_{\mathcal{A}}^{1} \\
\vdots \\
\operatorname{Tr} \rho_{\mathcal{A}} E_{\mathcal{A}}^{k}
\end{array}\right]+\left[\begin{array}{ccc}
e_{11} & \cdots & e_{1 k} \\
\vdots & \ddots & \vdots \\
e_{k 1} & \cdots & e_{k k}
\end{array}\right] \hat{\theta}^{t}
$$

or in a different notation

$$
\left[\begin{array}{c}
\varepsilon_{1} \\
\vdots \\
\varepsilon_{k}
\end{array}\right]=\left[\begin{array}{c}
e_{1}+\operatorname{Tr} \rho_{\mathcal{A}} E_{\mathcal{A}}^{1} \\
\vdots \\
e_{k}+\operatorname{Tr} \rho_{\mathcal{A}} E_{\mathcal{A}}^{k}
\end{array}\right]+T \hat{\theta}^{t}
$$

where $T$ is the $k \times k$ matrix from the previous formula. Therefore,

$$
\hat{\theta}^{t}=T^{-1}\left(\left[\begin{array}{c}
\varepsilon_{1} \\
\vdots \\
\varepsilon_{k}
\end{array}\right]-\left[\begin{array}{c}
e_{1}+\operatorname{Tr} \rho_{\mathcal{A}} E_{\mathcal{A}}^{1} \\
\vdots \\
e_{k}+\operatorname{Tr} \rho_{\mathcal{A}} E_{\mathcal{A}}^{k}
\end{array}\right]\right) .
$$

We have

$$
\hat{\theta}^{t}-\theta^{t}=T^{-1}\left(\left[\begin{array}{c}
\varepsilon_{1} \\
\vdots \\
\varepsilon_{k}
\end{array}\right]-\left[\begin{array}{c}
p_{1} \\
\vdots \\
p_{k}
\end{array}\right]\right) .
$$


The quadratic error matrix is the expected value of

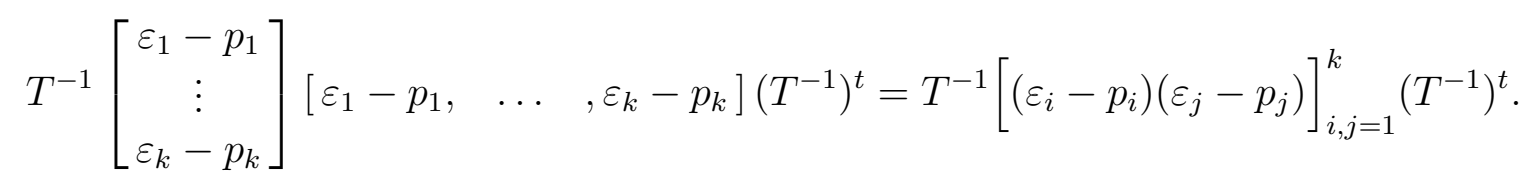

Due to the independence of the measurements the expected value of

$$
\left[\left(\varepsilon_{i}-p_{i}\right)\left(\varepsilon_{j}-p_{j}\right)\right]_{i, j=1}^{k}
$$

will be diagonal.

The random variables $\varepsilon_{i}$ have Bernoulli distribution and their variance is

$$
\left(1-\operatorname{Tr} \rho E^{i}\right) \operatorname{Tr} \rho E^{i} .
$$

We want to take the average:

$$
\int\left(1-\operatorname{Tr} \rho E^{i}\right) \operatorname{Tr} \rho E^{i} d \mu(\rho)
$$

where we integrate on the unitarily invariant states, and $\mu$ is the corresponding normalized Haar measure. For the sake of simplicity, assume that the operators $E^{i}$ have the same spectrum. Then the integral is constant: it does not depend on the actual $E_{i}$, so the average of the quadratic error matrix is

$$
c T^{-1}\left(T^{-1}\right)^{t}
$$

The determinant is minimal if the determinant of the matrix $T$ is maximal. Geometrically, the determinant is the volume of the parallelepiped determined by the row vectors. To maximize the determinant, the row vectors should be long. This implies that $E_{\mathcal{A}}^{i}=0$ in (11), since otherwise we could project $E^{i}$ on to $\mathbb{C} I \oplus \mathcal{B}$, and still have the same elements in $T$. So we have

$$
E^{i}=e+e_{i 1} F_{1}+\ldots+e_{i k} F_{k} \quad(1 \leq i \leq k),
$$

and then the determinant of the matrix

$$
\left[\begin{array}{cccc}
1 / n & 0 & \cdots & 0 \\
e & e_{11} & \cdots & e_{1 k} \\
\vdots & \vdots & \ddots & \vdots \\
e & e_{k 1} & \cdots & e_{k k}
\end{array}\right]
$$

is $\operatorname{det} T$. The angle of the first row and any other row is fixed. To have a large determinant the rows of $T$ should be orthogonal. In this case, the operators $E^{1}, \ldots, E^{k}$ are quasi-orthogonal. 
Theorem 1 If the positive contractions $E^{1}, \ldots, E^{k}$ have the same spectrum, then the determinant of the average of the quadratic error matrix is minimal if the operators $E^{1}, \ldots, E^{k}$ are complementary to each other and to $\mathcal{A}$.

Example 1 If two qubits are given and the reduced states of both qubits are known, then the ideal state estimation is connected to the observables

$$
\sigma_{11}, \sigma_{22}, \sigma_{33}, \sigma_{12}, \sigma_{23}, \sigma_{31}, \sigma_{13}, \sigma_{21}, \sigma_{32}
$$

where $\sigma_{i j}=\sigma_{i} \otimes \sigma_{j}$.

\section{The optimal POVM}

Once again we are in the n-dimensional case, but in this section we will use POVMs for state estimation. Assume that $\mathcal{A}=\{0\}$, which means that all parameters are unknown. Then

$$
E_{i}=e_{i} I+E_{\mathcal{B}}^{i}=e_{i}\left(I+f_{i} \cdot \sigma\right) \quad\left(1 \leq i \leq n^{2}\right),
$$

where $e_{i} \in \mathbb{R}, f_{i} \in \mathbb{R}^{n^{2}-1}, f_{i} \cdot \sigma=\sum_{j} f_{i j} \sigma_{j}$ and $\left\{\sigma_{j}: 1 \leq j \leq n^{2}-1\right\}$ are generalized Pauli matrices, $\operatorname{Tr} \sigma_{i}=0, \operatorname{Tr} \sigma_{i}^{2}=1, \operatorname{Tr} \sigma_{i} \sigma_{j}=0$. The positivity condition of $E_{i}$ is not known, but we have a necessary condition:

Lemma 1 If the $n \times n$ matrix $I+g \cdot \sigma$ is positive, then $\sum_{k} g_{k}^{2} \leq n^{2}-n$. If $\sum_{k} g_{k}^{2}=$ $n^{2}-n$, then $I+g \cdot \sigma=n P$ with a projection $P$ of rank 1 .

Proof. $A=g \cdot \sigma$ is self-adjoint, $\operatorname{Tr} A=0$. Let $\lambda_{1}, \lambda_{2}, \ldots, \lambda_{n}$ be the eigenvalues of $A$. Then

$$
\sum_{k=1}^{n^{2}-1} g_{k}^{2}=\operatorname{Tr} A^{2}=\sum_{t=1}^{n} \lambda_{t}^{2} .
$$

Since $\sum_{t=1}^{n} \lambda_{t}=0$ from $\operatorname{Tr} A=0$ and $\lambda_{t} \geq-1$ from $I+A \geq 0$, we have the upper bound. Namely, $\sum_{t=1}^{n} \lambda_{t}^{2}$ is maximal, if $\lambda_{1}, \lambda_{2}, \ldots, \lambda_{n}$ is a permutation of the numbers $-1,-1, \ldots,-1, n-1$. In this case $I+A$ has eigenvalues $0,0, \ldots, 0, n$, so it is a multiple of a projection.

The computations are similar to the von Neumann case. The probabilities of different outcomes are

$$
\left[\begin{array}{c}
p_{1} \\
\vdots \\
p_{d}
\end{array}\right]=\left[\begin{array}{c}
e_{1} \\
\vdots \\
e_{d}
\end{array}\right]+T\left[\begin{array}{c}
\theta_{1} \\
\vdots \\
\theta_{d}
\end{array}\right]
$$

where $d=n^{2}-1$ and $T=\left(e_{1}, e_{2}, \ldots, e_{d}\right)^{T}\left(f_{1}, f_{2}, \ldots, f_{d}\right)$ dyadic matrix. 
If $\nu_{1}, \nu_{2}, \ldots \nu_{d}$ are the relative frequencies of the outcomes (of different measurements on identical copies), then the estimator is

$$
\left[\begin{array}{c}
\hat{\theta}_{1} \\
\vdots \\
\hat{\theta}_{d}
\end{array}\right]=T^{-1}\left[\begin{array}{c}
\nu_{1}-e_{1} \\
\vdots \\
\nu_{d}-e_{d}
\end{array}\right]
$$

The mean quadratic error matrix is

$$
V(\theta)=T^{-1} W\left(T^{-1}\right)^{*}
$$

with $W$ denoting the covariance matrix of the random variables $\nu_{1}, \nu_{2}, \ldots \nu_{d}$. These are multinomially distributed, so

$$
W=\left[\begin{array}{cccc}
p_{1}\left(1-p_{1}\right) & -p_{1} p_{2} & \ldots & -p_{1} p_{d} \\
-p_{1} p_{2} & p_{2}\left(1-p_{2}\right) & \ldots & -p_{2} p_{d} \\
\vdots & \vdots & & \vdots \\
-p_{1} p_{d} & -p_{2} p_{d} & \ldots & p_{d}\left(1-p_{d}\right)
\end{array}\right]
$$

To obtain the average mean quadratic error matrix, we integrate $W$ on the rotation invariant states $H$ with respect to the normalized Haar measure $\mu$. We have to calculate two types of integrals:

$$
\begin{gathered}
\int_{H}-p_{i} p_{j} d \mu(\theta)=-\int_{H}\left(e_{i}+e_{i}\left\langle f_{i}, \theta\right\rangle\right)\left(e_{j}+e_{j}\left\langle f_{j}, \theta\right\rangle\right) d \mu(\theta) \\
=-\int_{H} e_{i} e_{j} d \mu(\theta)-e_{i} e_{j} \int_{H}\left\langle f_{i}, \theta\right\rangle d \mu(\theta)-\int_{H} e_{i} e_{j}\left\langle f_{j}, \theta\right\rangle d \mu(\theta)-e_{i} e_{j} \int_{H}\left\langle f_{i}, \theta\right\rangle\left\langle f_{j}, \theta\right\rangle d \mu(\theta) \\
=-e_{i} e_{j}+0+0-e_{i} e_{j} \alpha\left\langle f_{i}, f_{j}\right\rangle .
\end{gathered}
$$

The integrals in the middle are zeros because of the symmetry: $\int_{H}\langle v, \theta\rangle d \mu(\theta)=0$. The last integral comes from:

$$
\int_{H}\left\langle f_{i}, \theta\right\rangle\left\langle f_{j}, \theta\right\rangle d \mu(\theta)=\int_{H}\left(\sum_{\ell=1}^{d} f_{i, \ell} \theta_{\ell}\right)\left(\sum_{m=1}^{d} f_{j, m} \theta_{m}\right) d \mu(\theta)=\sum_{\ell=1}^{d}\left(\int_{H} \theta_{\ell}^{2} d \mu(\theta)\right) f_{i, \ell} f_{j, \ell}
$$

The quantity $\int_{H} \theta_{\ell}^{2} d \mu(\theta)$ does not depend on $\ell$ : it is a constant $\alpha$ depending on the domain of the integration; this way we obtained the stated formula.

Similarly,

$$
\int_{H} p_{i}\left(1-p_{i}\right) d \mu(\theta)=e_{i}\left(1-e_{i}\right)-e_{i}^{2} \alpha\left\langle f_{i}, f_{i}\right\rangle
$$

so we can calculate the average quadratic error matrix:

$$
F^{-1}\left[\begin{array}{cccc}
e_{1}^{-1}-1-\alpha\left\langle f_{1}, f_{1}\right\rangle & -1-\alpha\left\langle f_{1}, f_{2}\right\rangle & \cdots & -1-\alpha\left\langle f_{1}, f_{d}\right\rangle \\
-1-\alpha\left\langle f_{1}, f_{2}\right\rangle & e_{2}^{-1}-1-\alpha\left\langle f_{2}, f_{2}\right\rangle & \cdots & -1-\alpha\left\langle f_{2}, f_{d}\right\rangle \\
\vdots & \vdots & & \vdots \\
-1-\alpha\left\langle f_{1}, f_{d}\right\rangle & -1-\alpha\left\langle f_{2}, f_{d}\right\rangle & \cdots & e_{d}^{-1}-1-\alpha\left\langle f_{d}, f_{d}\right\rangle
\end{array}\right]\left(F^{-1}\right)^{*}
$$


where $F=\left(f_{1}, f_{2}, \ldots, f_{d}\right)$.

The minimizer should be symmetric, hence $e_{i}=1 / n^{2},\left\langle f_{i}, f_{i}\right\rangle=x$ and $\left\langle f_{i}, f_{j}\right\rangle=y$, if $i \neq j, i, j \leq n^{2}-1$. Then the determinant has the form $A / B$, where

$$
A=\left(n^{2}-\alpha(x-y)\right)^{n^{2}-2}\left(1-\alpha\left(x+\left(n^{2}-2\right) y\right)\right)
$$

and

$$
B=(x-y)^{n^{2}-2} \cdot\left(x+\left(n^{2}-2\right) y\right)
$$

Therefore, we minimize

$$
\frac{A}{B}=\left(\frac{n^{2}}{x-y}-\alpha\right)^{n^{2}-2}\left(\frac{1}{x+\left(n^{2}-2\right) y}-\alpha\right) .
$$

We can also calculate the length of $f_{n^{2}}=-\sum_{i=1}^{n^{2}-1} f_{i}$ :

$$
\left\langle f_{n^{2}}, f_{n^{2}}\right\rangle=\left(n^{2}-1\right) \cdot\left(x+\left(n^{2}-2\right) y\right) \leq n^{2}-n
$$

where the latter inequality is the condition for positivity, see Lemma 1. On the other hand, we have the condition

$$
x \leq n^{2}-n .
$$

If both inequalities were sharp, then (13) would not be minimal, because we could increase both $x$ and $y$ with a sufficiently small $\varepsilon$ and then the value of (13) would be smaller. If the equality holds in (14), then the second term of (13) will be constant. So we want to have the difference of $x$ and $y$ as large as possible, hence $x$ should be maximal (and then $y$ is minimal). If the equality holds in (15), then from (14) we have $y \leq-\left(n^{2}-n\right) /\left(n^{2}-1\right)$, and on this domain (13) has a negative derivative. So the minima are taken at

$$
x=n^{2}-n, \quad \text { and } \quad y=-\frac{n^{2}-n}{n^{2}-1} .
$$

Lemma 1 gives

$$
E_{i}=\frac{1}{n^{2}}\left(I+f_{i} \cdot \sigma\right)=\frac{1}{n} P_{i}
$$

with some projections $P_{i}$ and

$$
\operatorname{Tr} P_{i} P_{j}=\frac{1}{n^{2}}\left\langle I+f_{i} \sigma, I+f_{j} \sigma\right\rangle=\frac{1}{n}+\frac{1}{n^{2}} y=\frac{1}{n+1} .
$$

We arrived at a system (6). The following statement is obtained.

Theorem 2 If a symmetric informationally complete system exists, then the optimal POVM is described by its projections $P_{i}$ as $E_{i}=P_{i} / n\left(1 \leq i \leq n^{2}\right)$.

For a qubit the existence of the symmetric informationally complete POVM is obvious, there are other known examples in lower dimensions, but in $n$ dimensions the existence is not known. 


\section{A non-symmetrical qubit case for POVM}

In contrast to the previous section where we constructed an optimal POVM without any information on the state, in the upcoming part we will show some special, nontrivial cases, where some information is known. This is a much harder task, therefore the achieved results are not so strong, nevertheless, it is even more interesting than the fully unknown case.

Let us assume, first of all, that we have a qubit: $\theta_{1}, \theta_{2}$ are not known, but $\theta_{3}$ is given, so the density operator is only partially unknown. In this case the POVM will have three components $\left\{E_{1}, E_{2}, E_{3}\right\}$, the sufficient number of components to make the state estimation. Of course we can use a greater number of components but in that case the construction of the estimator is not trivial.

We use the parametrization

$$
E_{1}=a_{0}(I+a \cdot \sigma), \quad E_{2}=b_{0}(I+b \cdot \sigma), \quad E_{3}=c_{0}(I+c \cdot \sigma),
$$

where $a_{0}, b_{0}, c_{0} \in \mathbb{R}$, and $a, b, c$ are vectors in $\mathbb{R}^{3}$. From $E_{1}+E_{2}+E_{3}=I$, we have $a_{0}+b_{0}+c_{0}=1$ and $a_{0} a+b_{0} b+c_{0} c=0$. The positivity conditions are:

$$
0 \leq a_{0}, b_{0}, c_{0} \quad\|a\|,\|b\|,\|c\| \leq 1 .
$$

Similarly to the previous section we should minimize a determinant of the form $A / B$, where

$$
\begin{gathered}
A=\operatorname{det} V, \quad B=\operatorname{det}^{2}\left[\begin{array}{ll}
a_{1} & a_{2} \\
b_{1} & b_{2}
\end{array}\right]=\operatorname{det}\left[\begin{array}{cc}
\left\langle a^{*}, a^{*}\right\rangle & \left\langle a^{*}, b^{*}\right\rangle \\
\left\langle a^{*}, b^{*}\right\rangle & \left\langle b^{*}, b^{*}\right\rangle
\end{array}\right]=: \operatorname{det} C, \\
V=\left[\begin{array}{cc}
p a_{0}^{-1}-p^{2} & -p q \\
-p q & q b_{0}^{-1}-q^{2}
\end{array}\right]-\beta\left(1-\theta_{3}^{2}\right)\left[\begin{array}{cc}
\left\langle a^{*}, a^{*}\right\rangle & \left\langle a^{*}, b^{*}\right\rangle \\
\left\langle a^{*}, b^{*}\right\rangle & \left\langle b^{*}, b^{*}\right\rangle
\end{array}\right]=: D-c \cdot C \\
p=1+a_{3} \theta_{3}, \quad q=1+b_{3} \theta_{3}, \quad a^{*}=\left(a_{1}, a_{2}\right), \quad b^{*}=\left(b_{1}, b_{2}\right),
\end{gathered}
$$

where $\beta$ is a constant depending on the particular unitarily invariant surface of the integration, $0 \leq \beta \leq 1$, so $c$ is a given constant on interval $\left[0,1-\theta_{3}^{2}\right]$.

For the minimizer we can assume some symmetry conditions: $a_{0}=b_{0}=c_{0}=\frac{1}{3}$, and we will minimize $A$ and maximize $B$ independently to obtain the optimum:

$$
\begin{gathered}
A=\left(d_{11}-c c_{11}\right)\left(d_{22}-c c_{22}\right)-\left(d_{12}-c c_{12}\right)^{2} \rightarrow \min , \\
B=c_{11} c_{22}-\left(c_{12}\right)^{2} \rightarrow \max .
\end{gathered}
$$

Let us suppose first that $a_{3}$ and $b_{3}$ are given (then the elements of $D$ are constants) and we want to optimize the other variables.

We know that

$$
\langle a+b, a+b\rangle=c_{11}+c_{22}+2 c_{12}+\left(a_{3}+b_{3}\right)^{2} \leq 1
$$


If $c_{12} \geq 0$, then from (18) we have $c_{11}+c_{22} \leq 1$, hence $B \leq 1 / 4$.

If $c_{12}<0$, then $B$ is maximal if $c_{12}$ is maximal, from (18) we have an upper bound:

$$
c_{12} \leq \frac{1-\left(a_{3}+b_{3}\right)^{2}-c_{11}-c_{22}}{2} .
$$

Substituting this upper bound in (17) we have to maximize it in $c_{11}$ and $c_{22}$. Using derivation we can obtain that it is maximal if $c_{11}$ and $c_{22}$ are maximal:

$$
c_{11}=a_{1}^{2}+a_{2}^{2} \leq 1-a_{3}^{2} \quad \text { and } \quad c_{22}=b_{1}^{2}+b_{2}^{2} \leq 1-b_{3}^{2} .
$$

Substituting this upper bound in (17) we get

$$
B=\frac{3}{4}-a_{3}^{2}-a_{3} b_{3}-b_{3}^{2}
$$

which is optimal if $a_{3}=b_{3}=0$. Then $B=3 / 4$, so it is a global optimum.

Lemma 2 The following inequality is always true:

$$
d_{12}-c c_{12} \leq 0
$$

Proof. We have

$$
d_{12}-c c_{12}=-\left(1+\theta_{3} a_{3}\right)\left(1+\theta_{3} b_{3}\right)-c\left(a_{1} b_{1}+a_{2} b_{2}\right) .
$$

Since $c \leq 1-\theta_{3}^{2}$ and

$$
a_{1} b_{1}+a_{2} b_{2}=\left\langle a^{*}, b^{*}\right\rangle \geq-\left\|a^{*}\right\|\left\|b^{*}\right\| \geq-\sqrt{1-a_{3}^{2}} \sqrt{1-b_{3}^{2}},
$$

so it is enough to show that

$$
\left(1+\theta_{3} a_{3}\right)\left(1+\theta_{3} b_{3}\right) \geq\left(1-\theta_{3}^{2}\right) \sqrt{1-a_{3}^{2}} \sqrt{1-b_{3}^{2}} .
$$

The right-hand side does not depend on the signs, the left-hand side is minimal if $\theta_{3} a_{3} \leq 0, \theta_{3} b_{3} \leq 0$, so it suffices to prove for positive $a_{3}, b_{3}, \theta_{3}$ that

$$
\left(1-\theta_{3} a_{3}\right)\left(1-\theta_{3} b_{3}\right) \geq\left(1-\theta_{3}^{2}\right) \sqrt{1-a_{3}^{2}} \sqrt{1-b_{3}^{2}}
$$

This is true since from the Cauchy-Schwarz inequality follows that

$$
a_{3} \theta_{3}+\sqrt{1-a_{3}^{2}} \sqrt{1-\theta_{3}^{2}} \leq 1 \quad \Longrightarrow \quad 1-a_{3} \theta_{3} \geq \sqrt{1-a_{3}^{2}} \sqrt{1-\theta_{3}^{2}}
$$

and a similar statement is true for $b_{3}$. 
Using this lemma, we get that $A$ is minimal if $c_{12}$ is maximal, and from there the solution is almost the same as in the previous case, we only have more complicated calculations. We substitute the upper bound (19) in to (16) and we can obtain that it is minimal if $c_{11}$ and $c_{22}$ are maximal. Using the bounds (20) we get for $A$ a function of $a_{3}$ and $b_{3}$; using differentiation we can obtain that $a_{3}=b_{3}=0$ gives the optimal solution here, too.

So in both cases we have equality in (20), so $\left\langle a^{*}, a^{*}\right\rangle=\left\langle b^{*}, b^{*}\right\rangle=1$ and equality in (19), so $\left\langle a^{*}, b^{*}\right\rangle=-1 / 2$. Since $\|a\|=\|b\|=1$,

$$
\frac{3}{2} E_{1}=\frac{1}{2}(I+a \cdot \sigma), \quad \frac{3}{2} E_{2}=\frac{1}{2}(I+b \cdot \sigma)
$$

are projections. We have $\|c\|=\|a+b\|=1$ and this implies that $3 E_{3} / 2$ is a projection as well.

Theorem 3 The optimal POVM for the unknown $\theta_{1}$ and $\theta_{2}$ can be described by projections $P_{i}(i=1,2,3)$ :

$$
E_{i}=\frac{2}{3} P_{i}, \quad \sum_{i=1}^{3} P_{i}=\frac{3}{2} I, \quad \operatorname{Tr} \sigma_{3} P_{i}=0, \quad \operatorname{Tr} P_{i} P_{j}=\frac{1}{4} \quad \text { for } \quad i \neq j .
$$

The optimal POVM is quasi-orthogonal to the subalgebra generated by $\sigma_{3}$ and symmetric in the other directions. This result is in some sense the combination of Theorem 1 and Theorem 2, with complementarity and symmetricalness both occurring in the same problem. The conditions of (자) are valid, but with different constants than in the SIC-POVM case.

It is an interesting question whether the existence of a conditional SIC-POVM in Theorem 3 is just a coincidence and it follows from the geometrical structure of $M_{2}(\mathbb{C})$, or there are such objects in higher dimensions. The following example solves this problem:

Example 2 Let the elements of a POVM in $M_{3}(\mathbb{C})$ be

$$
\begin{gathered}
E_{1}=\frac{1}{7}\left[\begin{array}{lll}
1 & 1 & 1 \\
1 & 1 & 1 \\
1 & 1 & 1
\end{array}\right], \quad E_{2}=\frac{1}{7}\left[\begin{array}{ccc}
1 & \varepsilon^{6} & \varepsilon^{2} \\
\varepsilon & 1 & \varepsilon^{3} \\
\varepsilon^{5} & \varepsilon^{4} & 1
\end{array}\right], \quad E_{3}=\frac{1}{7}\left[\begin{array}{ccc}
1 & \varepsilon^{2} & \varepsilon^{3} \\
\varepsilon^{5} & 1 & \varepsilon \\
\varepsilon^{4} & \varepsilon^{6} & 1
\end{array}\right], \\
E_{4}=\frac{1}{7}\left[\begin{array}{ccc}
1 & \varepsilon^{4} & \varepsilon^{6} \\
\varepsilon^{3} & 1 & \varepsilon^{2} \\
\varepsilon & \varepsilon^{5} & 1
\end{array}\right], \quad E_{5}=\overline{E_{2}}, \quad E_{6}=\overline{E_{3}}, \quad E_{7}=\overline{E_{4}},
\end{gathered}
$$

where $\varepsilon=\exp (2 \pi \mathrm{i} / 7)$. This POVM fulfills the conditions in (6) with constants $k=7$, $\lambda=7 / 3$ and $\mu=2 / 9$. Moreover it is quasi-orthogonal to diagonal matrices, so it is a conditional SIC-POVM with respect to the diagonal matrices. 
It is still an open question, however, that exactly in which cases a conditional SICPOVM exists. Furthermore, does it minimize the determinant of the average covariance matrix? The answer to the first question is definitely a difficult problem, because it contains the question of the existence of SIC-POVMs. Our conjecture to the second question is that conditional SIC-POVMs are optimal if they exist, and so they can play an important role in quantum information theory, but an exhaustive investigation exceeds the scope of this paper.

\section{Discussion and conclusions}

A density matrix $\rho \in M_{n}(\mathbb{C})$ has $n^{2}-1$ real parameters. If there are no known parameters, several two-valued POVMs $\left\{F_{1}, I-F_{1}\right\},\left\{F_{2}, I-F_{2}\right\}, \ldots,\left\{F_{n^{2}-1}, I-F_{n^{2}-1}\right\}$ or a single POVM $\left\{E_{1}, E_{2}, \ldots, E_{n^{2}}\right\}$ are considered. The estimator can be constructed as a linear function of the outcomes, and since they are multinomially and binomially distributed, their variance can be calculated easily. By averaging them on the invariant states and taking the determinant of the average covariant matrix, we will get comparable results. The optimum is at the quasi-orthogonal measurements (Theorem 1) and at a symmetric informationally complete system (Theorem 2), respectively. If partial information is known, the concept is the same as previously, but as there are some known parameters, the domain of integration is restricted. From Theorem 1, it turns out that in the von Neumann case the optimal measurements are not only complementary to each other, but to the known subalgebras as well. For POVMs a qubit case is described in detail: conditionally symmetric informationally complete systems turn out to be optimal. The latter is a new concept, an example for the qutrit case is obtained too, further investigations are suggested.

\section{References}

[1] L. Accardi, Some trends and problems in quantum probability, in Quantum probability and applications to the quantum theory of irreversible processes, eds. L. Accardi, A. Frigerio and V. Gorini, Lecture Notes in Math. 1055, 1-19. Springer, 1984 .

[2] E. Bagan, M.A. Ballester, R.D. Gill, A. Monras and R. Munoz-Tapia, Optimal full estimation of qubit mixed states, Phys. Rev. A, 73, 032301, 2006.

[3] T. Baier and D. Petz, Complementarity and state estimation, Rep. Math. Phys., 65, 203-214, 2010.

[4] G. M. D'Ariano, M. G. A. Paris, and M. F. Sacchi, Quantum tomography, Advances in Imaging and Electron Physics, 128, 205-308, 2003 
[5] G. M. D'Ariano, M. F. Sacchi and J. Kahn, Minimax quantum state discrimination, Phys. Rev. A, 72, 032310, 2005.

[6] R. Demkowicz-Dobrzanski, Beyond quantum Fisher information: optimal phase estimation with arbitrary a priori knowledge, Phys. Rev. A, 83, 061802, 2011.

[7] G. M. D'Ariano, P. Lo Presti and P. Perinotti, Classical randomness in quantum measurements, J. Phys. A: Math. Gen., 38, 5979-5991, 2005

[8] D.G. Fisher and M. Freyberger, Estimating mixed quantum states, Physics Letters A, 273, 293-302, 2000.

[9] K. Kraus, Complementary observables and uncertainty relations. Phys. Rev. D (3) 35, 3070-3075, 1987.

[10] M. Hayashi and K. Matsumoto, Asymptotic performance of optimal state estimation in quantum two level system, J. Math. Phys. 49, 102101, 2008.

[11] C. W. Helstrøm, Quantum decision and estimation theory, Academic Press, New York, 1976.

[12] T. Konrad, O. Gühne, J. Audretsch and H. J. Briegel, Parameter estimation for mixed states from a single copy, Phys. Rev. A, 75, 062101, 2007.

[13] R. L. Kosut, I. Walmsley and H. Rabitz, Optimal experiment design for quantum state and process tomography and Hamiltonian parameter estimation, arXiv, quant-ph/0411093, 2004

[14] H. Ohno, D. Petz and A. Szántó, Quasi-orthogonal subalgebras of $4 \times 4$ matrices, Linear Alg. Appl. 425, 109-118, 2007.

[15] S. Olivares and M. G. A. Paris, Quantum estimation via minimum Kullback entropy principle, Phys. Rev. A, 76, 042120, 2007.

[16] J. Oppenheim, K. Horodecki, M. Horodecki, P. Horodecki and R. Horodecki, A new type of complementarity between quantum and classical information, Phys. Rev. A 68, 022307, 2003.

[17] D. Petz, Quantum Information Theory and Quantum Statistics, Springer-Verlag, Heidelberg, 2008.

[18] D. Petz, K.M. Hangos, A. Szántó and F. Szöllösi, State tomography for two qubits using reduced densities, J. Phys. A, 39, 10901-10907, 2006.

[19] D. Petz, K.M. Hangos and A. Magyar, Point estimation of states of finite quantum systems, J. Phys. A, 40, 7955-7969, 2007.

[20] D. Petz, K.M. Hangos and L. Ruppert, Quantum state tomography with finite sample size, in Quantum Bio-Informatics, eds. L. Accardi, W. Freudenberg, M. Ohya, World Scientific, pp. 247-257, 2008. 
[21] D. Petz, Complementarity in quantum systems, Rep. Math. Phys. 59, 209-224, 2007.

[22] D. Petz, Algebraic complementarity in quantum theory, J. Math. Phys. 51, 015215, 2010 .

[23] D. Petz and J. Kahn, Complementary reductions for two qubits, J. Math. Phys., 48, 012107, 2007.

[24] D. Petz, A. Szántó and M. Weiner, Complementarity and the algebraic structure of 4-level quantum systems, J. Infin. Dim. Analysis Quantum Prob. 12, 99-116, 2009 .

[25] J. Rehacek, B.-G. Englert and D Kaszlikowski, Minimal qubit tomography, Physical Review A, 70, 052321, 2004.

[26] M. Renes, R. Blume-Kohout, A. J. Scott and C. M. Caves, Symmetric informationally complete quantum measurements, J. Math. Phys. 45, 2171, 2004.

[27] M. B. Ruskai, Some connections between frames, mutually unbiased bases, and POVM's in quantum information theory, Acta Appl. Math. 108, 709-719, 2009.

[28] A. J. Scott, Tight informationally complete quantum measurements, J. Phys. A 39, 13507, 2006.

[29] W.K. Wootters and B.D. Fields, Optimal state determination by mutually unbiased measurements, Ann. Physics, 191, 363-381, 1989. 